\title{
Pollen Number and Ribosome Gene Expression Altered in a Genome-Editing Mutant of REDUCED POLLEN NUMBER1 Gene
}

\author{
Hiroyuki Kakui 1,2,3, Takashi Tsuchimatsu ${ }^{1,4,5,6,7}$, Misako Yamazaki1, \\ Masaomi Hatakeyama ${ }^{1,8}$ and Kentaro K. Shimizu1,2,4*
}

${ }^{1}$ Department of Evolutionary Biology and Environmental Studies, University of Zurich, Zurich, Switzerland, ${ }^{2}$ Kihara Institute for Biological Research, Yokohama City University, Yokohama, Japan, ${ }^{3}$ Graduate School of Science and Technology, Niigata University, Niigata, Japan, ${ }^{4}$ Department of Plant and Microbial Biology \& Zurich-Basel Plant Science Center, University of Zurich, Zurich, Switzerland, ${ }^{5}$ Gregor Mendel Institute, Austrian Academy of Sciences, Vienna BioCenter, Vienna, Austria, ${ }^{6}$ Department of Biology, Chiba University, Chiba, Japan, ${ }^{7}$ Department of Biological Sciences, University of Tokyo, Tokyo, Japan, ${ }^{8}$ Functional Genomics Center Zurich, Zurich, Switzerland

\section{OPEN ACCESS}

Edited by:

David Honys,

Institute of Experimental Botany of the Czech Academy of Sciences, Czechia

Reviewed by: Gorou Horiguchi, Rikkyo University, Japan Etienne Delannoy,

UMR 9213 Institut des Sciences des Plantes de Paris Saclay (IPS2), France

*Correspondence: Kentaro K. Shimizu kentaro.shimizu@uzh.ch

Specialty section: This article was submitted to Plant Development and EvoDevo, a section of the journal Frontiers in Plant Science

Received: 31 August 2021 Accepted: 08 December 2021 Published: 11 January 2022

Citation:

Kakui H, Tsuchimatsu T, Yamazaki M, Hatakeyama M and Shimizu KK (2022) Pollen Number and Ribosome Gene Expression Altered in a Genome-Editing Mutant of REDUCED POLLEN NUMBER 1 Gene. Front. Plant Sci. 12:768584. doi: 10.3389/fp/s.2021.768584
The number of pollen grains varies within and between species. However, little is known about the molecular basis of this quantitative trait, in contrast with the many studies available on cell differentiation in the stamen. Recently, the first gene responsible for pollen number variation, REDUCED POLLEN NUMBER1 (RDP1), was isolated by genome-wide association studies of Arabidopsis thaliana and exhibited the signature of natural selection. This gene encodes a homolog of yeast Mrt4 (mRNA turnover4), which is an assembly factor of the large ribosomal subunit. However, no further data were available to link ribosome function to pollen development. Here, we characterized the RDP1 gene using the standard $A$. thaliana accession Col-0. The frameshift mutant, rdp1-3 generated by CRISPR/Cas9 revealed the pleiotropic effect of RDP1 in flowering, thus demonstrating that this gene is required for a broad range of processes other than pollen development. We found that the natural Col-0 allele conferred a reduced pollen number against the Bor-4 allele, as assessed using the quantitative complementation test, which is more sensitive than transgenic experiments. Together with a historical recombination event in Col-0, which was identified by sequence alignment, these results suggest that the coding sequence of RDP1 is the candidate region responsible for the natural phenotypic variation. To elucidate the biological processes in which RDP1 is involved, we conducted a transcriptome analysis. We found that genes responsible for ribosomal large subunit assembly/biogenesis were enriched among the differentially regulated genes, which supported the hypothesis that ribosome biogenesis is disturbed in the rdp1-3 mutant. Among the pollen-development genes, three key genes encoding basic helix-loop-helix (bHLH) transcription factors (ABORTED MICROSPORES (AMS), bHLH010, and bHLH089), as well as direct downstream genes of $A M S$, were downregulated in the rdp1-3 mutant. In summary, our results suggest a specialized function of ribosomes in pollen development through RDP1, which harbors natural variants under selection.

Keywords: pollen number, REDUCED POLLEN NUMBER1, complementation test, CRISPR/Cas9, transcriptome, ribosomal protein 


\section{INTRODUCTION}

Pollen grain number in seed plants is a key reproductive trait that has been studied extensively for decades, from agricultural and evolutionary viewpoints. Domesticated crop species generally produce fewer pollen grains than wild relative species, whereas a high pollen number is preferred in some cultivation strategies, such as hybrid crops and artificial pollination (Oka and Morishima, 1967; Franco-Mora et al., 2005; Langer et al., 2014; Shimizu and Tsuchimatsu, 2015; Boeven et al., 2016). Despite its agricultural and evolutionary importance, the genetic basis of pollen grain number has remained elusive, mainly because of its quantitative nature. Recently, the REDUCED POLLEN NUMBER1 (RDP1) gene, which is responsible for the natural variation in pollen number, was identified through a genomewide association study of Arabidopsis thaliana (Tsuchimatsu et al., 2020). The CRISPR/Cas9-generated RDP1 frameshift mutants in the Col-0 background produced about half of the number of pollen grains compared with wild-type and was considered non-functional or null. The Uod-1 accession had a long-haplotype variant at the RDP1 region, which conferred a lower number of pollen grains and exhibited a signature of selective sweep. The Bor-4 accession had an alternative variant that conferred a greater number of pollen grains. Both variants did not contain a gene disruptive mutation. Causal evidence of the allelic effects of RDP1 was obtained using Uod-1 and Bor-4 via the quantitative complementation test (also known as reciprocal hemizygosity test) (Tsuchimatsu et al., 2020), which compares allelic effects under the same copy number and positions of genes (Stern, 1998, 2014; Turner, 2014). Although functional tests using transgenesis are often used to examine exact causal mutations, a subtle allelic effect may not be detected in this manner because of the uncertainty of the insertion position in the genome in plants. Further quantitative complementation testing using an accession that experienced historical recombination events (such as Col-0) is another experiment that can narrow down the specific region responsible for the allelic effect of RDP1.

In contrast with pollen-number regulation, the molecular basis of cell differentiation in stamen development has been well studied (Alvarez-Buylla et al., 2010; Walbot and Egger, 2016; Ferguson et al., 2017; Li et al., 2017; Xue et al., 2021). Pollen and tapetal cell lineages differentiate from archesporial cells, with the latter being essential for the development of the former (Sanders et al., 1999; Feng and Dickinson, 2010; Walbot and Egger, 2016). The development of tapetum and pollen lineages is governed by a genetic pathway called "DYT1-TDF1-AMS-MS188-MS1" (Li et al., 2017), which is composed of transcriptional factors (Zhu et al., 2011; Lou et al., 2018). Two of them, DYSFUNCTIONAL TAPETUM1 (DYT1) and ABORTED MICORSPORES (AMS), are basic helixloop-helix (bHLH) transcription factors that interact with three

Abbreviations: AMS, ABORTED MICROSPORES; bHLH, basic helix-loop-helix; DEG, differentially expressed gene; DYT1, DYSFUNCTIONAL TAPETUM1; FAA, formaldehyde acetic acid; FDR, false discovery rate; GO, gene ontology; Mrt4, mRNA turnover4; RDP1, REDUCED POLLEN NUMBER1. other bHLH factors, bHLH10, bHLH89, and bHLH91. Their protein interactions and transcriptional feedback regulations were reported (Feng et al., 2012; Zhu et al., 2015; Cui et al., 2016). Among these bHLH factors, AMS is known as a master regulator of pollen wall formation (Sorensen et al., 2003; Xu et al., 2010; Zhu et al., 2011; Xu et al., 2014). Transcriptome data showed that many genes are differentially expressed between wild-type and ams mutant. Li et al. (2017) detected 825 downregulated genes from tapetum cells in the ams mutant. Xu et al. (2014) reported 23 genes that were directly regulated by AMS based on microarray and qChIPPCR experiments. Based on these detailed characterizations of transcriptional regulation and protein interaction in the pollen/anther pathway (Zhu et al., 2011; Ma et al., 2012; Cui et al., 2016; Ferguson et al., 2017; Wang et al., 2018; Chen et al., 2019), we investigated these pollen development genes in the mutants of $R D P 1$.

RDP1 encodes a protein with homology to the mRNA turnover 4 (Mrt4) protein. Mrt4 acts as a pre-60S ribosomal component in yeast (Schmidt et al., 2013; Salih et al., 2020; Tsuchimatsu et al., 2020). The Mrt4 gene is not essential in yeast, but null mutants of mrt4 show slow-growth phenotypes, suggesting its role in cell proliferation (Rodriguez-Mateos et al., $2009 \mathrm{a}, \mathrm{b})$. However, it is unclear whether RDP1 is working as a component of ribosome and affect the translation in A. thaliana. Ribosomal genes were traditionally considered housekeeping genes of the protein synthesis machinery, and to function uniformly in all cells. Nevertheless, increasing evidence has shown ribosome specialization in plant and other model species (Martinez-Seidel et al., 2020; Norris et al., 2021). Many mutants of ribosome-related genes exhibit organ specific phenotypes, such as the reduction of leaf cell number, larger leaf cell size (Fujikura et al., 2009; Horiguchi et al., 2011), reduced root length (Creff et al., 2010), and reduction of stamen number (Stirnberg et al., 2012). Transcriptome studies of ribosome biogenesis factor mutants of Arabidopsis reported the upregulation of ribosomal genes as well as altered regulation of other genes, which supported the importance of transcriptome data of ribosome biogenesis factors mutant (Beine-Golovchuk et al., 2018; Cheong et al., 2021).

Here, we focused on the standard Col-0 background for further characterization of the RDP1 gene. First, the phenotypes at different developmental stages were examined in the frameshift rdp1-3 mutant in the Col-0 background. Second, the RDP1 genomic sequences of Col-0, Uod-1, and Bor- 4 were aligned to characterize the Col- 0 sequence, and the quantitative complementation test was used to narrow down the candidate regions responsible for the natural phenotypic variation. Subsequently, we performed transcriptome analyses to detect differentially expressed genes (DEGs) between the wild-type and rdp1-3 plants focusing on ribosome-related and pollen development genes. Finally, we assessed whether ribosome genes were upregulated, as previously observed in Arabidopsis ribosome mutants. Our data support the specific function of the ribosome in the developing pollen cell lineage. 


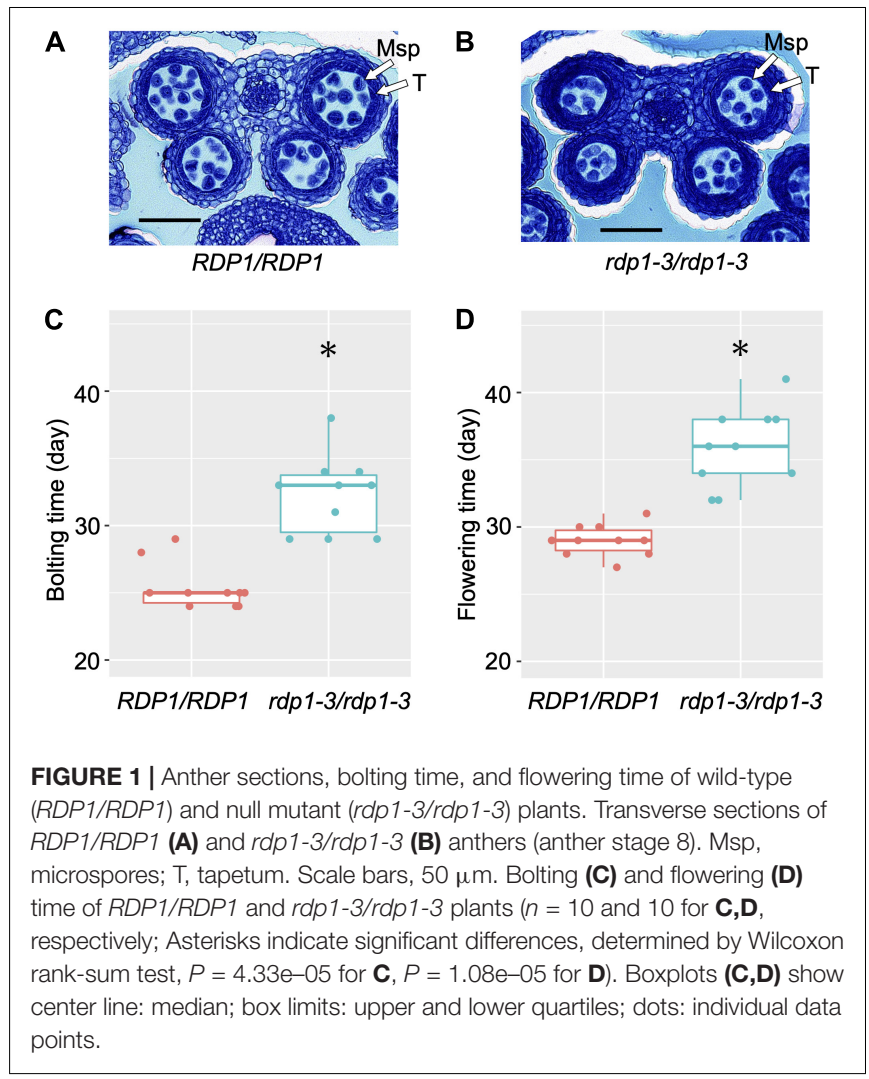

\section{MATERIALS AND METHODS}

\section{Plant Materials and Growth Conditions}

Four accessions of $A$. thaliana, Col-0 (N22625), Bor-4 (N22591), Mz-0 (N22636), and Uod-1 (N22612), as well as A. lyrata ssp. lyrata (CS22696), were used in this study. Arabidopsis seeds were sown on soil mixed with the insecticide ActaraG (Syngenta Agro, Switzerland) and stratified for 3-4 days at $4^{\circ} \mathrm{C}$ in the dark. The plants were grown under $16 \mathrm{~h}$ of light at $22^{\circ} \mathrm{C}$ and $8 \mathrm{~h}$ of dark at $20^{\circ} \mathrm{C}$, with weekly treatments with insecticide (Kendo Gold, Syngenta Agro). The frameshift mutants of RDP1 created by CRISPR/Cas9, i.e., $r d p 1-3$ (Col-0) and $r d p 1-6$ (Bor-4), were generated previously (Tsuchimatsu et al., 2020). The frameshift of $r d p 1-3$ and $r d p 1-6$ were identical. These frameshift mutants were genotyped by PRIMA using primers (Forward primer, 5'-TAGGCACAATGGAAAGTTAG-3'; Reverse primer, 5'-TT AACATAAAAGAACCATTGTAAG-3') and 40-mer probe $\left(5^{\prime}-\right.$ GGAGACTTTGTAGATACCAGAGTTCATCTTCTGCAGAAC G-3') as described previously (Kakui et al., 2021). T-DNA fragments containing CRISPR/Cas9 and GFP marker were removed from each strain of $r d p 1-3$ and $r d p 1-6$ by selecting non-fluorescence seeds (Tsutsui and Higashiyama, 2017). Bolting time was determined when the main inflorescence stem reached $3 \mathrm{~cm}$. Flowering time was recorded when the first flower opened. Time-lapse movie of plant growth from Col-0 and rdp1-3 was obtained using a TLC200 Pro camera (Brinno, Taiwan).

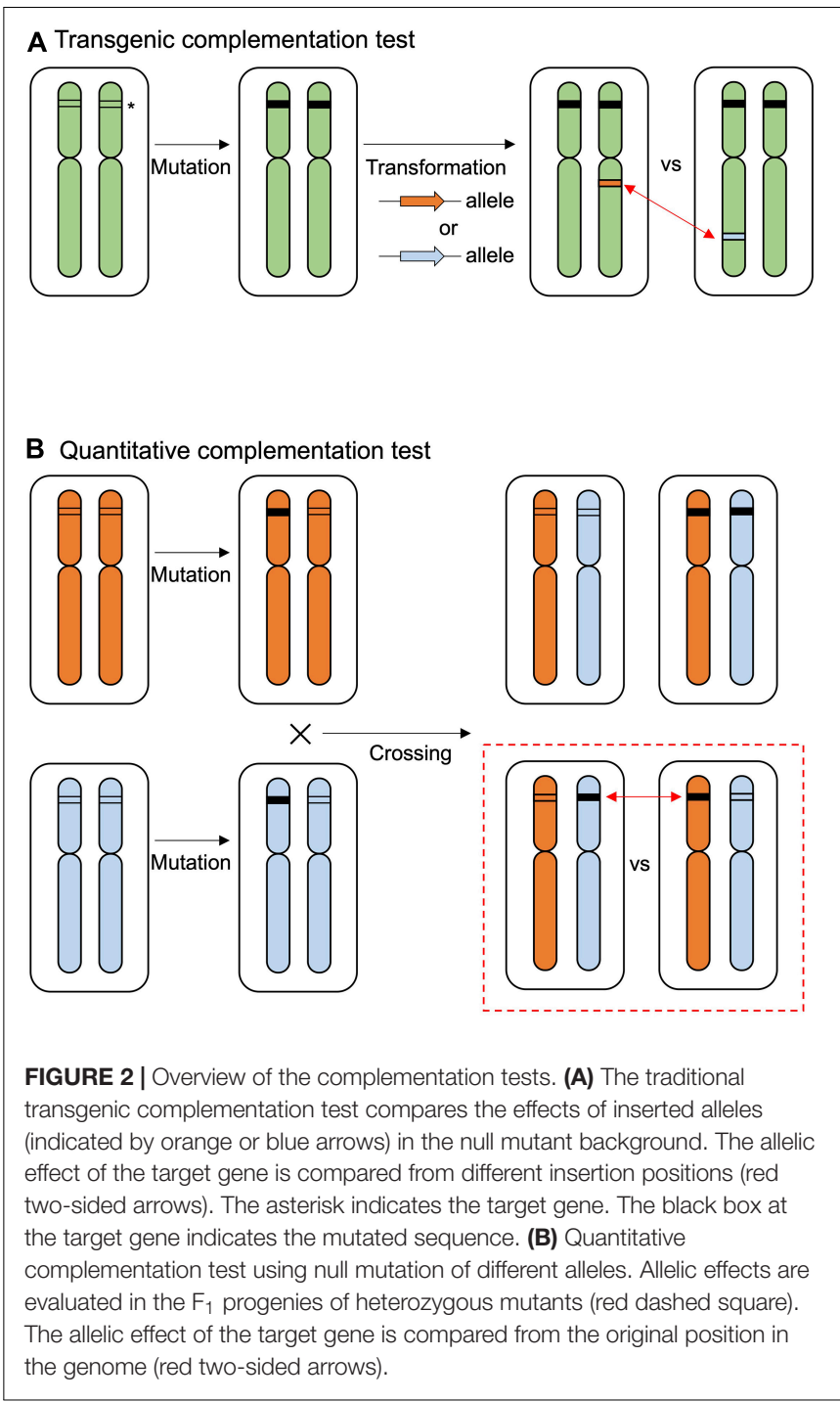

\section{Histological Analysis of Anthers}

For histological analysis, inflorescences were fixed with FAA (formaldehyde:acetic acid:70\% ethanol = 1:1:18), dehydrated, and embedded in Technovit 7100 according to the manufacturer's instructions (Heraeus Kulzer GmbH, Wehrheim, Germany). Five-micrometer sections were cut with a microtome (RM2145, Leica, Germany) and stained with toluidine blue before observation under a Leica microscope (DM5000, Leica) equipped with a color camera (DMC2900, Leica).

\section{Traditional Transgenic Complementation}

The RDP1 sequence of Col-0, including the 1,960 bp upstream region from the coding sequence to $661 \mathrm{bp}$ downstream from the stop codon, was amplified using the following primers: 3795_Atlg25260F $\left(5^{\prime}-\right.$ TTTCTCCCCACATTTCTC-3' $)$ and 3988_Atlg25260R (5'-TATGTTATCAAAATTCATAAAATG$\left.3^{\prime}\right)$. The $R D P 1$ sequences from different accessions were amplified from Mz-0, Col-0, Bor-4, and A. lyrata by PCR (PrimeSTAR GXL, Takara Bio, Japan). These PCR products were cloned 

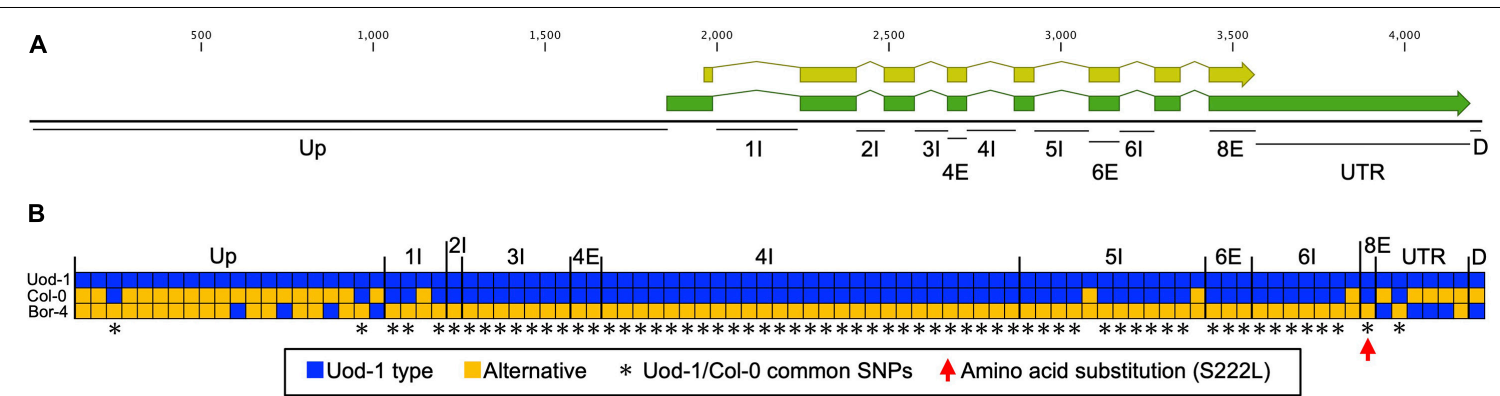

C

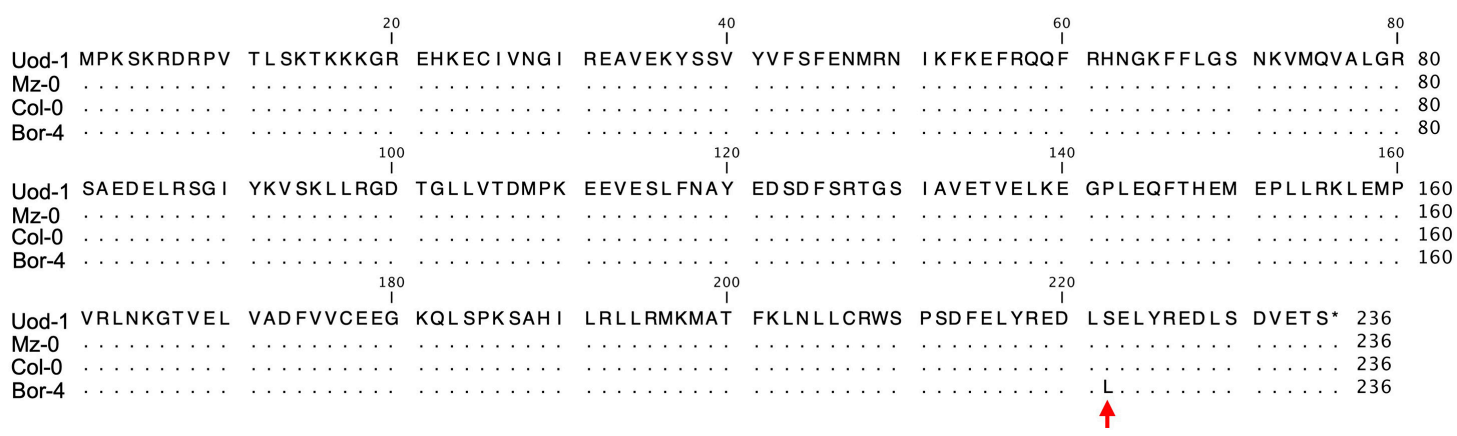

FIGURE 3 | Comparison of RDP1 sequences among Uod-1, Col-0, and Bor-4. (A) Schematic structure of the RDP1 gene. The green and yellow boxes indicate mRNA and coding regions, respectively. Up, upstream; I, intron; E, exon; UTR, 3'UTR; D, downstream. Each annotation was defined by NCBI information (NM_102335.4). The number with I or E stands for the ordinal number of each intron or exon. Only the names of polymorphic regions are indicated. (B) Haplotype map of the RDP1 gene. All SNPs and gaps (indels) of the RDP1 region are shown. Each box corresponds to a polymorphism. Continuous gaps (indels) are displayed as a single box. The blue box indicates the Uod-1 type, and the orange box indicates an alternative polymorphism. The asterisks indicate common SNPs between Uod-1 and Col-0. The red arrow indicates an amino acid substitution in RDP1 (S222L). The nucleic acid sequences of the RDP1 region corresponding to (A) region from Uod-1, Col-0, and Bor-4 are shown in Supplementary Figure 1. (C) Amino acid sequences of RDP1 from Uod-1, Mz-0, Col-0, and Bor-4. Dots represent identical sequence with Uod-1. Single amino acid substitution alone was identified among these accessions (S222L, red arrow). Sequence annotations and multiple sequence alignment were generated using the CLC Main Workbench (version 21.0.5)(A,C, and Supplementary Figure 1).

into pFAST-R01 (Shimada et al., 2010). Each construct was independently transformed into $r d p 1-3$ (Col-0 background) plants using the floral-dip method (Clough and Bent, 1998) with Agrobacterium tumefaciens (GV3101).

\section{Quantitative Complementation}

Arabidopsis thaliana plants with heterozygous $R D P 1$ alleles from two accessions, i.e., the Col-0 accession (RDP1/rdp1-3, termed $R D P 1^{\mathrm{Col}} / r d p 1^{\mathrm{Col}}$ hereafter) and the Bor-4 accession (RDP1/rdp1-6, termed $R D P 1^{\text {Bor }} / r d p 1^{\text {Bor }}$ hereafter), were prepared (Tsuchimatsu et al., 2020). $F_{1}$ plants were generated by crossing $R D P 1^{\mathrm{Col}} / r d p 1^{\mathrm{Col}}$ and $R D P 1^{\mathrm{Bor}} / r d p 1^{\text {Bor }}$. Subsequently, $\mathrm{F} 1$ progenies are genotyped and analyzed.

\section{Pollen-Number Counting Using a Cell Counter}

We sampled flower buds and counted pollen numbers using a cell counter as described previously (Kakui et al., 2020; Tsuchimatsu et al., 2020). In summary, flowers of stage 12 [unopened anther with mature pollen grains (Smyth et al., 1990)] were collected and incubated overnight at $60^{\circ} \mathrm{C}$. Flowers were collected from the side stem because flowers of the main stem have significantly larger pollen numbers (Tsuchimatsu et al., 2020). 1st and 2 nd flowers of flower buds were excluded from sampling because they tend to show abnormal flower shapes (Kakui et al., 2020). We collected flowers from the start of flowering until approximately 2 weeks later. Subsequently, $30 \mu \mathrm{L}$ of $5 \%$ Tween-20 was added, and the mixture was sonicated using a sonicator (Bioruptor Plus, Diagenode, Belgium), to release the pollen grains. The pollen suspension was mixed with a pollen counting solution (CASYton, OMNI Life Science, Germany), and particles were counted on a cell counter (CASY cell counter, OMNI Life Science). Pollen-number data were statistically analyzed and plots were constructed in $\mathrm{R}$ (R Core Team, 2013).

\section{Transcriptome Analysis}

Total RNA was isolated from four replicates of flower buds of the wild-type and $r d p 1-3$ plants using the RNeasy plant mini kit (Qiagen, Germany). The samples encompassed a wide range of developmental stages (flower stages 1-12) (Sanders et al., 1999), excluding opened flowers. Next, 75-bp single-end read sequencing was performed on a NextSeq 500 sequencer (Illumina, San Diego, CA, United States). The sample information and the number of reads are listed in Supplementary Table 1. The transcriptome data obtained were analyzed using the SUSHI framework (Hatakeyama et al., 2016). We defined DEGs based on a false discovery rate $(F D R)<0.1$. All DEGs data 

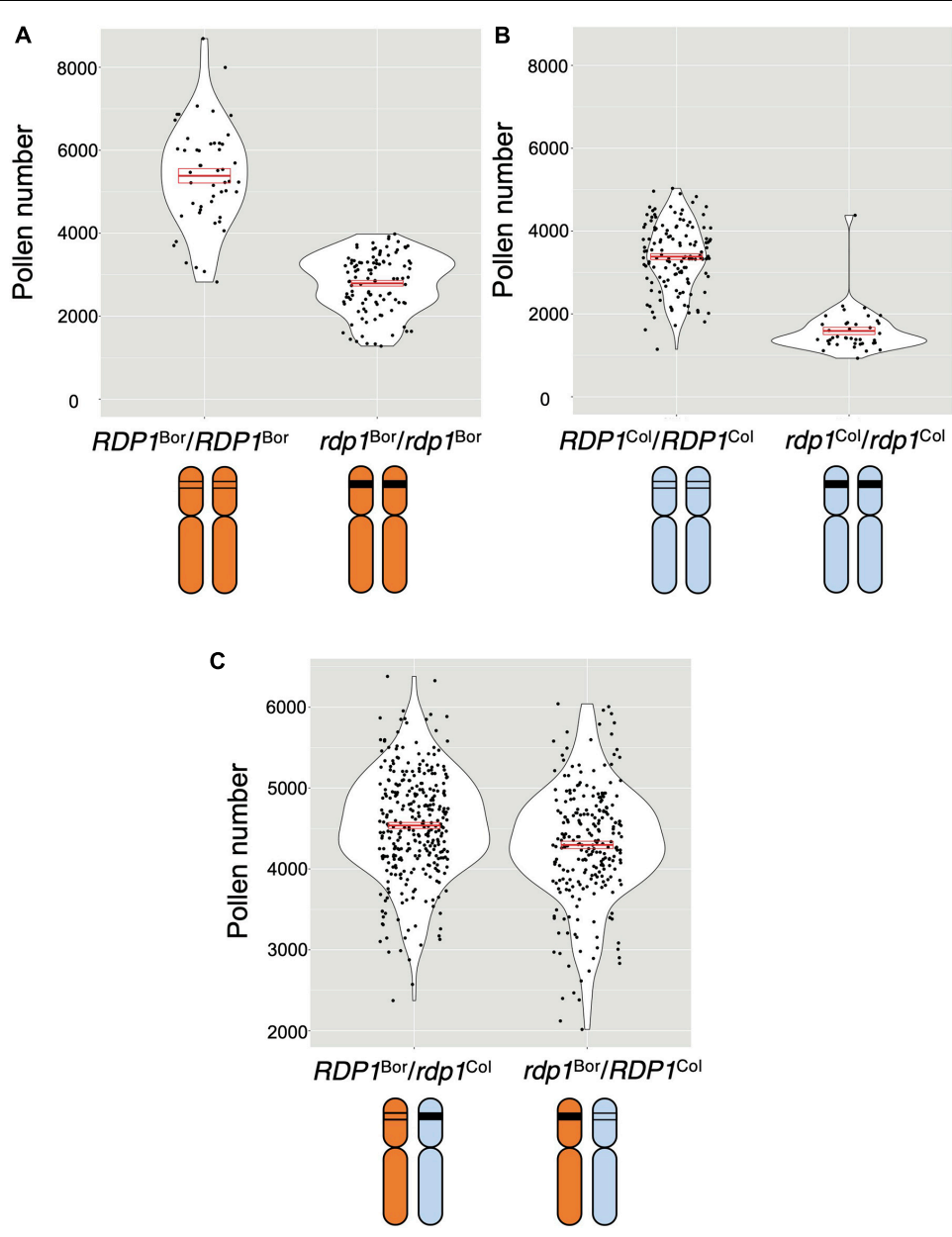

FIGURE 4 | Quantitative complementation test of the RDP1 gene. Pollen number per flower was analyzed. Violin plots with means and standard errors of means indicated by red bold bars and boxes, respectively. (A,B) Pollen-number difference between the wild-type and a homozygote of a frameshift allele generated by the CRISPR/Cas9 technique in the Bor-4 background (A; $\left.n=50\left[R D P 1^{\text {Bor }} / R D P 1^{\text {Bor }}\right] ; n=106\left[r_{1 p} 1^{\text {Bor }} / \mathrm{rdp}^{\text {Bor }}\right]\right)$ and in the Col-0 background $(\mathbf{B} ; n=135$ $\left.\left[R D P 1^{\mathrm{Col}} / R D P 1^{\mathrm{Col}}\right] ; n=40\left[r d p 1_{\mathrm{Col}} / r d p 1^{\mathrm{Col}}\right]\right)$. (C) Difference in the effect of two natural alleles, $R D P 1^{\text {Bor }}$ and $R D P 1^{\mathrm{Col}}$, on pollen number. The pollen number in the plants with $R D P 1^{\text {Bor }}$ was significantly higher than that in the plants with $R D P 1^{\mathrm{Col}}$ (nested analysis of variance; $P=1.10 \times 10^{-5} ; n=315\left[R D P 1^{\mathrm{Bor}} / \mathrm{rdp}^{\mathrm{Col}}\right.$, median $=4,528$ pollen grains/flower]; $n=247$ [rdp $1^{\text {Bor }} / R D P 1^{\mathrm{Col}}$, median $=4,302$ pollen grains/flower]). The two alleles were compared in the heterozygous state with a frameshift CRISPR/Cas9 allele in an identical genomic background. $\mathrm{F}_{1}$ plants were obtained from the cross of two heterozygotes, RDP1 Col/rdp 1 Col and $R D P 1^{\text {Bor }} / r d p 1^{\text {Bor }}$. Results from four genotypes including $R D P 1^{\text {Bor }} / R D P 1^{\text {Col }}$ and $r d p 1^{\text {Bor } / r d p}$ 1 Col were shown in Supplementary Figure 3.

are listed in Supplementary Table 2. The gene ontology (GO) enrichment analysis was performed using the ShinyGO v0.66 software (Ge et al., 2020).

\section{RESULTS}

\section{Natural Variation of RDP1 Sequences and the Validation of Allelic Functional Differences Using the Quantitative Complementation Test}

The CRISPR/Cas9-generated frameshift $r d p 1-3$ mutant in the Col-0 background produced about half the number of pollen grains compared with the wild-type counterpart (Tsuchimatsu et al., 2020). We further observed the section of the developing anther and confirmed that the number of microspores was already decreased in the mutant at the anther stage 8 (Sanders et al., 1999; Figures 1A,B). Tsuchimatsu et al. (2020) reported that the frameshift $r d p 1-3$ mutants exhibited other pleiotropic phenotypes, such as reduced ovule number and delayed growth, in contrast with the effect of natural variants. Here, we found that the bolting and flowering times were significantly delayed (Wilcoxon ranksum test; $P=4.33 \mathrm{e}-05$ for bolting time, $P=1.08 \mathrm{e}-05$ for flowering time, Figures 1C,D and Supplementary Video 1). These results support the notion that RDP1 functions as an Mrt4 homolog in A. thaliana, in accordance with the slower growth observed in the Mrt4 null mutant in yeast (Rodriguez-Mateos et al., 2009a).

To narrow down the causal sequence/region underlying the allelic effect of $R D P 1$, we performed functional experiments 
using natural accessions. We first performed a traditional transgenic test to detect the allelic effect of RDP1 (Figure 2A), but found that the resolution was not sufficient to detect it, as follows. The prepared RDP1 sequences ranged from the upstream region (1,960 bp in Col-0) to the downstream region (661 bp in Col-0, Supplementary Figure 1) of three A. thaliana accessions (Col-0, Mz-0, and Bor-4), as well as A. lyrata ssp. lyrata. Mz-0 was among the accessions with the lowest pollen number, and the predominantly outcrossing species A. lyrata produced a much greater number of pollen grains than the A. thaliana accessions (Tsuchimatsu et al., 2020). Each sequence was introduced into the CRISPR/Cas9-generated $r d p 1$ null (nonfunctional) mutant ( $r d p 1-3 / r d p 1-3$, Col-0 background), and more than 10 independent transformants were obtained for each of the four constructs. However, the variation in pollen number was high, even among the transformants of the same construct, and the range of the number of pollen grains largely overlapped between the four constructs (Supplementary Figures 2A,B). When these data were compared with those obtained for wildtype Col-0, three of them showed no significant difference, whereas, unexpectedly, the construct with Col-0 showed a slight increase in pollen number, although a previous complementation test on another $r d p 1$ mutant background ( $r d p 1-1)$ showed no significant difference compared with Col-0 (Tsuchimatsu et al., 2020). We speculate that the positional effect of the insertion site was too large to detect a subtle allelic difference. In Arabidopsis transgenic experiments, it is difficult to control the insertion sites of transgenes. Depending on the insertion position, the expression level, timing, and/or tissue type of the transgenes may be affected and other genes can be disrupted. Although a drastic increase in the number of independent transgenic lines may eventually result in significant differences, the construction of these lines and the measurement of phenotypes would be highly tedious.

We then used the quantitative complementation test to detect the allelic effect of RDP1. Previously, we showed the allelic differences of $R D P 1$ between Uod-1 (a variant conferring reduced pollen number) and Bor-4 (a variant conferring increased pollen number) via the quantitative complementation test (Figure 2B; Tsuchimatsu et al., 2020). Here, we tested quantitative complementation using the Bor-4 and Col-0 accessions. We aligned the RDP1 genomic region of Col-0 to that of Uod1 and Bor-4 (Figure 3A and Supplementary Figure 1). The analysis of the alignment of genomic sequences showed that Col0 experienced a historical recombination event near the start of the transcribed region, i.e., between its upstream and transcribed (exon/intron) regions (Figure 3B; Tsuchimatsu et al., 2020). The upstream sequence of Col-0 was close to that of Bor-4. However, the exonic and intronic sequences of Col-0 were very close to those of Uod-1, and there was only a single amino acid substitution differentiating Bor-4 from the three other accessions (S222L, Figures 3B,C). This amino acid position was deduced to be functionally important because the corresponding serine residues at the $\mathrm{C}$-terminal region of human Mrt4 regulate cellular localization under the stress condition (Michalec-Wawiorka et al., 2015). In addition, the 3'UTR and downstream regions of Col-0 had several unique substitutions

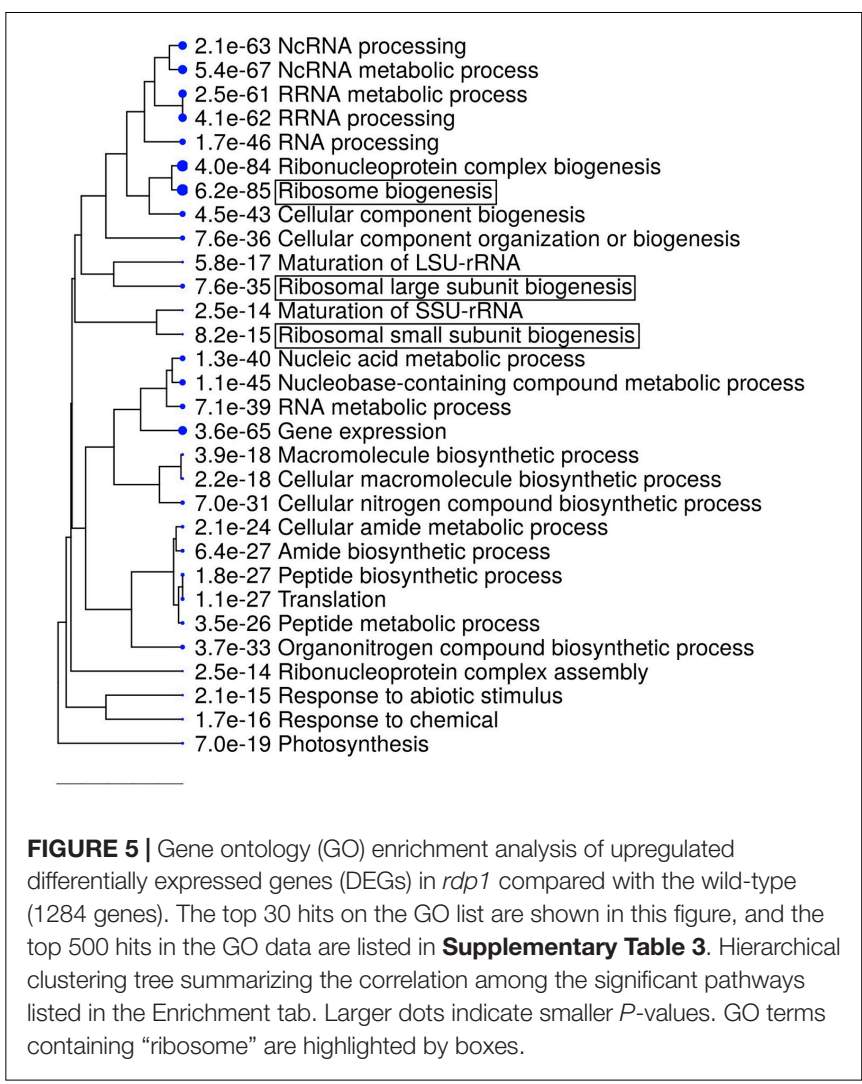

compared with Uod-1 and Bor-4. Phenotypically, Col-0 and Uod1 had smaller numbers of pollen grains (Col-0, 3,355 pollen grains/flower; Uod-1, 3,277 pollen grains/flower) in contrast with Bor-4 (4,528 pollen grains/flower) (Tsuchimatsu et al., 2020). The unique sequence character of Col-0 prompted us to study whether the coding region has a variant conferring reduced pollen number.

To compare the RDP1 allele, plants were prepared as follows (Figure 2B): (1) RDP1 heterozygous plants ( $R D P 1 / r d p 1)$ were prepared from the Bor-4 and Col-0 accessions; (2) two heterozygous plants were crossed; and (3) $R D P 1^{\mathrm{Bor}} / r d p 1^{\mathrm{Col}}$ and $r d p 1^{\mathrm{Bor}} / R D P 1^{\mathrm{Col}}$ plants were selected. These plants had a single different functional RDP1 allele in the original chromosomal position, but all other genome sequences were identical: a single disrupted $R D P 1$ allele with the same frameshift position and the same genome constitution (one chromosome from Bor-4 and the other from Col-0) outside of RDP1. We found that the plants with the functional Bor-4 allele of $R D P 1\left(R D P 1^{\text {Bor }} / r d p 1^{\mathrm{Col}}\right)$ had a significantly higher pollen number than those with a functional Col-0 allele $\left(r d p 1^{\mathrm{Bor}} / R D P 1^{\mathrm{Col}}\right)\left(P=1.10 \times 10^{-5}\right.$; Figure 4). We estimated allelic effect of $R D P 1$ based on median in the quantitative complementation test. $R D P 1^{\text {Bor }}$ is 1,599 pollen grain increasing $\left[R D P 1^{\mathrm{Bor}} / r d p 1^{\mathrm{Col}}(4,528\right.$ pollen grain) vs. $r d p 1^{\mathrm{Bor}} / r d p 1^{\mathrm{Col}}(2,929$ pollen grain $\left.)\right]$ and $R D P 1^{\mathrm{Col}}$ is 1,373 pollen grain increasing $\left[r d p 1^{\mathrm{Bor}} / R D P 1^{\mathrm{Col}}(4,302\right.$ pollen grain) vs. $r d p 1^{\mathrm{Bor}} / r d p 1^{\mathrm{Col}}(2,929$ pollen grain)] (Supplementary Figure 3). This result elucidated the allelic difference between Bor-4 and Col-0. 
TABLE 1 | DEGs from ribosomal small and large subunit-related genes (FDR $<0.1$ ).

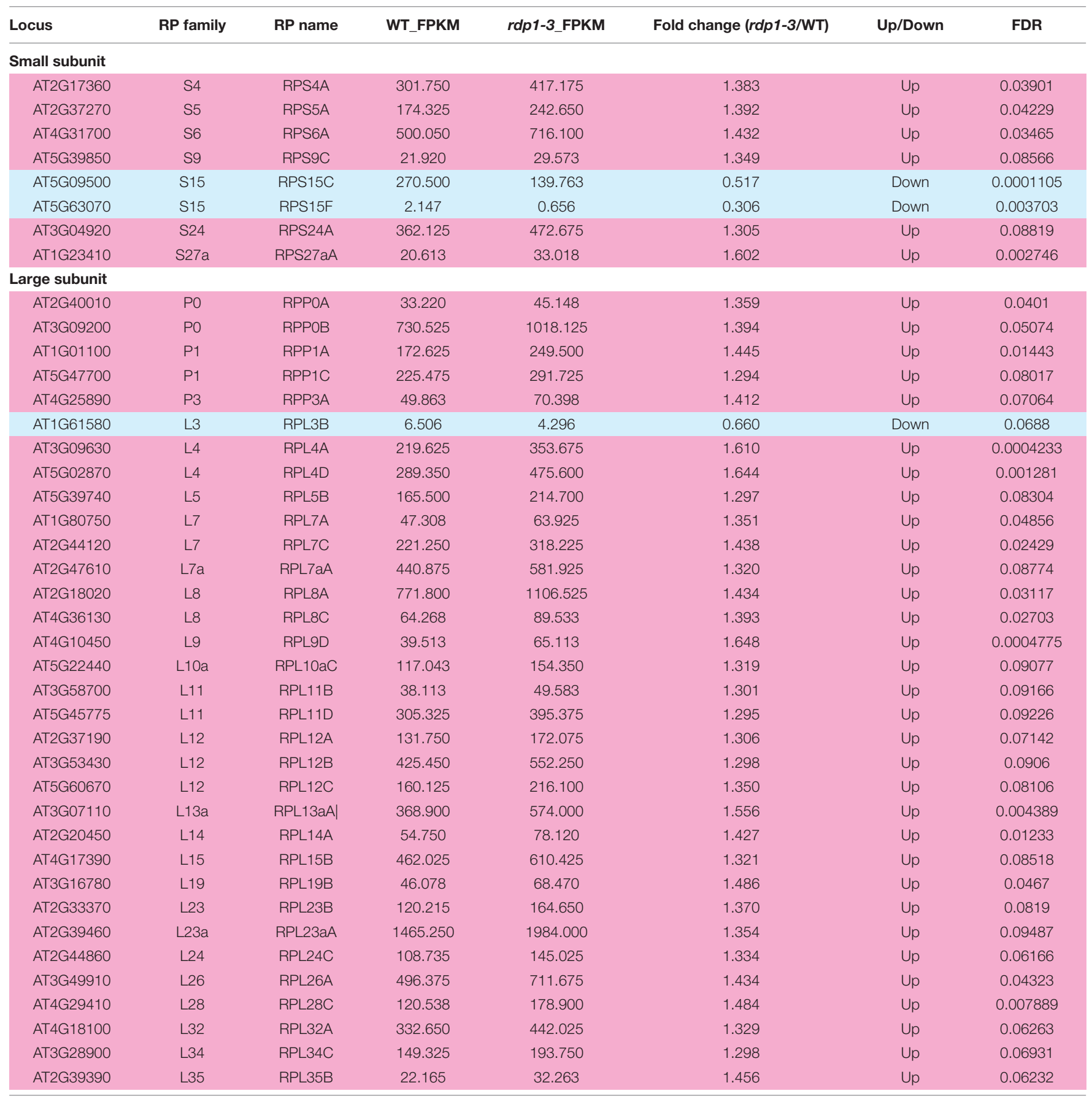

The pink and blue highlights indicate gene upregulation and downregulation, respectively. The list of cytosolic ribosomal genes was taken from previous reports (Barakat et al., 2001; Hummel et al., 2015).

\section{Enhanced Transcription of \\ Ribosome-Related Genes and the Reduction of That of Specific Anther/Pollen Development Genes in rdp1-3}

Next, we examined which biological processes are disturbed by the disruption of the RDP1 gene in flower bud tissues, and whether the expression of ribosome-related genes is altered as previously described for other Arabidopsis ribosome mutants. We performed a transcriptome analysis using wild-type of Col- 0 and rdp1-3 plants. A large number of DEGs between the wild-type and $r d p 1-3$ plants was detected using the criterion of FDR $<0.1$ (3,020 genes, Supplementary Table 2). Among them, 1,284 genes were upregulated and 1,736 genes were downregulated in $r d p 1-3$ plants. A GO term analysis showed the enrichment 
of many ribosome-related genes in the upregulated gene set (Figure 5 and Table 1 and Supplementary Table 3). These results strongly support the notion that $R D P 1$ of $A$. thaliana functions in the biogenesis of the ribosome as a yeast Mrt4 homolog (Lo et al., 2010; Michalec-Wawiorka et al., 2015; Greber, 2016; Saez-Vasquez and Delseny, 2019).

The GO term analysis also revealed the enrichment of genes relevant for pollen development, as well as pollenrelated categories in the downregulated gene set (e.g., "Pollen development," "Pollen wall assembly," and "Pollen tube growth"; Figure 6 and Supplementary Table 4). Among the genes relevant for pollen development, three bHLH transcription factor genes, bHLH010, bHLH089, and AMS, were downregulated (Table 2), all of which interact with the DYT1 during anther development (Feng et al., 2012; Ferguson et al., 2017). Although their fold change itself was modest (29.7-34.4\% reduction; Table 2 ), we also observed a systematic downregulation of genes that were directly regulated by AMS ( 19 out of $23, P=2.901 \mathrm{e}-15$, Fisher's exact test; Table 2; Xu et al., 2014). These data suggest that the bHLH pathway and its downstream genes were downregulated in the $r d p 1-3$ mutant. By contrast, we did not find any enrichment of GO terms related to ovule development, although ovule number was also reduced in the $r d p 1-3$ (Tsuchimatsu et al., 2020). This expression analysis in the flower bud suggests a distinctive role for RDP1 in anther development through transcription factor regulation.

\section{DISCUSSION}

\section{Quantitative Complementation Tests of Multiple Combinations to Narrow Down the Genomic Regions Responsible for Pollen-Number Regulation}

Although the quantitative complementation test has been validated in several animal and bacterial species, such as yeast, Drosophila, mosquito, fish, and mouse (Steinmetz et al., 2002; Stern, 2014; Turner, 2014; Podobnik et al., 2020), the preparation of mutants in a specific site of a gene represents a bottleneck regarding the application of quantitative complementation tests in plants (Stern, 2014). Recent progress in genome-editing technologies has allowed the efficient generation of frameshift non-functional mutants at the targeted gene in several different accessions. Thus, a quantitative complementation test will have a broader application of obtaining causal evidence of subtle allelic effects in plant species.

In this study, we performed a quantitative complementation test of RDP1 between Col-0 and Bor-4. A key of the method is to generate equivalent non-functional alleles in two different backgrounds by CRISPR/Cas9. We generated frameshift mutants at the same position, i.e., $r d p 1-3$ and $r d p 1-6$ in Col0 and Bor-4, respectively. We consider them as null mutants because frameshift mutants are in general disruptive, and the yeast studies suggested the importance of C-terminal domains (Michalec et al., 2010), but we note that the quantitative complementation test can work even if they are not completely

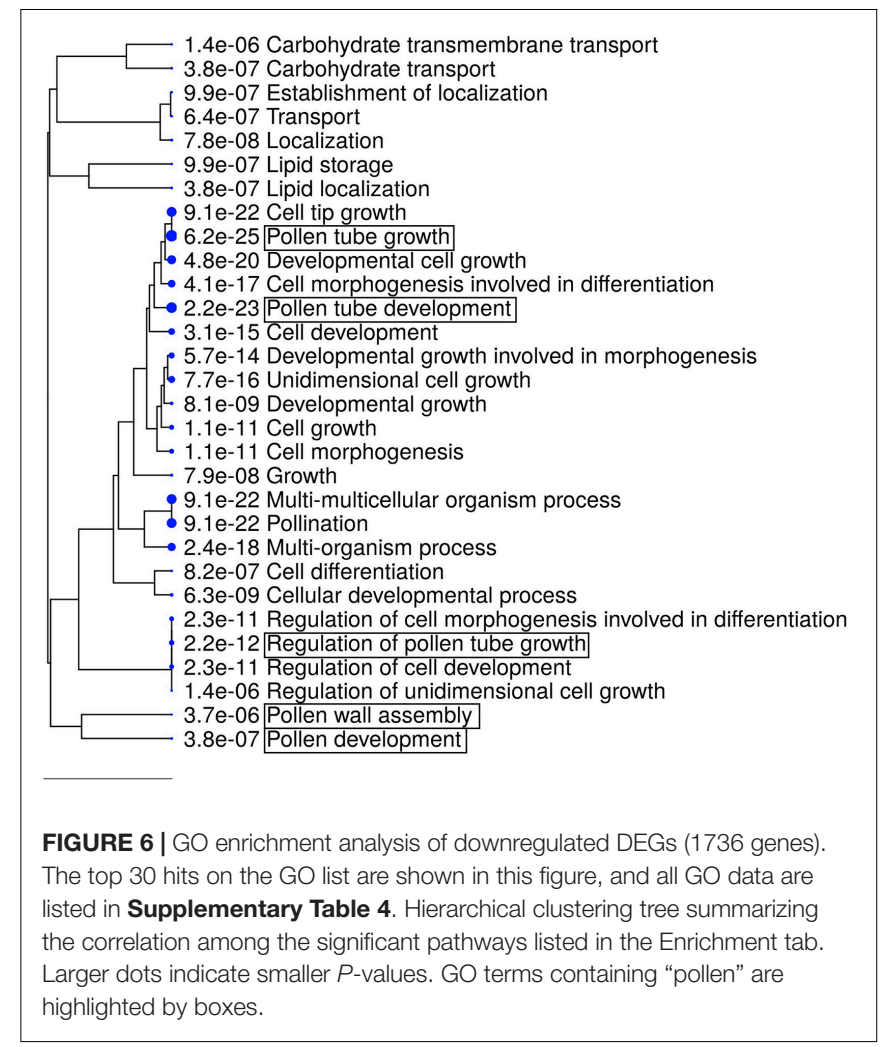

null. Combined with the previously reported results of the quantitative complementation test between Uod-1 and Bor-4 (Tsuchimatsu et al., 2020), our data support the notion that both Col-0 and Uod-1 have alleles that confer reduced pollen number against Bor-4. Although it is formally possible that more than two functional variants exist, the selective sweep signature of the long-haplotype variants suggests that a single mutation conferring reduced pollen number spread to many accessions, including Col-0 and Uod-1. This implies that the causal mutation of the reduced pollen number is shared between Col-0 and Uod-1, in contrast with Bor-4. The alignment of the genomic sequences of the three accessions revealed that such substitutions were mostly found in exon/intron regions, and not in upstream or downstream regions, reflecting the historical recombination inferred in Col-0. Although it is possible that mutations outside of the aligned region may have contributed to this phenomenon, substitutions in the exon/intron regions, including S222L, are promising candidate causal mutations of the reduced pollen number (Figures 3B,C), which warrants further experimental verification. In short, quantitative complementation tests using multiple accessions serve as a tool to narrow down the candidate regions of causal mutations of quantitative traits when transgenic experiments are not sufficiently powerful.

\section{RDP1 and the Biogenesis of the Ribosomal Large Subunit}

Remarkably, we detected two ontologies related to the ribosomal large subunit ("Ribosomal large subunit 
TABLE 2 | Downregulated pollen/anther development-related genes.

\begin{tabular}{|c|c|c|c|c|c|c|c|}
\hline ID & Gene symbol & Gene name/description & \multicolumn{2}{|c|}{ FPKM } & $\frac{\text { Fold change }}{(\text { rdp1-3/WT) }}$ & Up/Down & FDR \\
\hline AT4G27420 & ABCG9 & ATP-BINDING CASSETTE G9 & 10.36 & 6.73675 & 0.650 & Down & 0.03768 \\
\hline AT3G13220 & ABCG26 & ATP-BINDING CASSETTE G26 & 32.95 & 24.17 & 0.734 & Down & 0.07096 \\
\hline AT3G21180 & ACA9 & AUTOINHIBITED CA(2 +)-ATPASE 9 & 13.565 & 5.79125 & 0.427 & Down & 0.001642 \\
\hline AT1G69540 & AGL94 & AGAMOUS-LIKE 94 & 4.50075 & 2.462 & 0.547 & Down & 0.01472 \\
\hline AT1G22130 & AGL104 & AGAMOUS-LIKE 104 & 21.21 & 10.585 & 0.499 & Down & 0.006843 \\
\hline AT5G14380 & AGP6 & ARABINOGALACTAN PROTEIN 6 & 695.075 & 212.25 & 0.305 & Down & 0.0004339 \\
\hline AT2G16910 & AMS & ABORTED MICROSPORES & 20.7625 & 13.3375 & 0.642 & Down & 0.009175 \\
\hline AT5G54470 & BBX29 & B-BOX DOMAIN PROTEIN 29 & 1.01833 & 0.44235 & 0.434 & Down & 0.05074 \\
\hline AT5G59030 & COPT1 & COPPER TRANSPORTER 1 & 126.868 & 77.21 & 0.609 & Down & 0.03737 \\
\hline AT1G01280 & CYP703A2 & CYTOCHROME P450, FAMILY 703, SUBFAMILY A, POLYPEPTIDE 2 & 26.2025 & 18.22 & 0.695 & Down & 0.06944 \\
\hline AT3G04620 & DAN1 & D NUCLDUO1-ACTIVATEEIC ACID BINDING PROTEIN 1 & 50.7075 & 29.4975 & 0.582 & Down & 0.002855 \\
\hline AT3G60460 & DUO1 & DUO POLLEN 1 & 2.4205 & 1.26045 & 0.521 & Down & 0.07064 \\
\hline AT4G10950 & GELP77 & GDSL-TYPE ESTERASE/LIPASE 77 & 2.621 & 1.49225 & 0.569 & Down & 0.07858 \\
\hline AT5G54800 & GPT1 & GLUCOSE 6-PHOSPHATE/PHOSPHATE TRANSLOCATOR 1 & 55.88 & 36.22 & 0.648 & Down & 0.02275 \\
\hline AT4G22600 & INP1 & INAPERTURATE POLLEN1 & 1.95575 & 0.9254 & 0.473 & Down & 0.06207 \\
\hline AT1G19180 & JAZ1 & JASMONATE-ZIM-DOMAIN PROTEIN 1 & 36.1125 & 22.34 & 0.619 & Down & 0.01786 \\
\hline AT5G11110 & KNS2 & KAONASHI 2 & 6.718 & 4.33975 & 0.646 & Down & 0.02011 \\
\hline AT2G35210 & MEE28 & MATERNAL EFFECT EMBRYO ARREST 28 & 5.495 & 2.2425 & 0.408 & Down & 0.01798 \\
\hline AT4G14080 & MEE48 & MATERNAL EFFECT EMBRYO ARREST 48 & 445.275 & 249.95 & 0.561 & Down & 0.00152 \\
\hline AT5G05850 & PIRL1 & PLANT INTRACELLULAR RAS GROUP-RELATED LRR 1 & 19.8625 & 11.5435 & 0.581 & Down & 0.02079 \\
\hline AT4G34850 & PKSB & POLYKETIDE SYNTHASE B & 95.7175 & 67.7175 & 0.707 & Down & 0.09108 \\
\hline AT4G29470 & PLA2-DELTA & PHOSPHOLIPASE A2 DELTA & 5.387 & 1.73778 & 0.323 & Down & 0.005243 \\
\hline AT4G29460 & PLA2-GAMMA & PHOSPHOLIPASE A2 GAMMA & 0.65675 & 0.07614 & 0.116 & Down & 0.004069 \\
\hline AT5G39400 & PTEN1 & $\begin{array}{l}\text { PHOSPHATASE AND TENSIN HOMOLOG DELETED ON } \\
\text { CHROMOSOME TEN } 1\end{array}$ & 3.12565 & 0.68463 & 0.219 & Down & 0.09549 \\
\hline AT4G20050 & QRT3 & QUARTET 3 & 68.4625 & 43.4175 & 0.634 & Down & 0.004464 \\
\hline AT4G17530 & RAB1C & RAB GTPASE HOMOLOG $1 \mathrm{C}$ & 70.8125 & 46.1975 & 0.652 & Down & 0.01514 \\
\hline AT5G40260 & RPG1 & RUPTURED POLLEN GRAIN1 & 12.3375 & 7.75825 & 0.629 & Down & 0.04411 \\
\hline AT5G50800 & RPG2 & RUPTURED POLLEN GRAIN 2 & 19.1675 & 6.2265 & 0.325 & Down & 0.0006463 \\
\hline AT1G09180 & SAR1 & SECRETION-ASSOCIATED RAS 1 & 4.53925 & 2.48025 & 0.546 & Down & 0.06193 \\
\hline AT1G06515 & ssSPTa & SMALL SUBUNIT OF SPT A & 68.015 & 44.575 & 0.655 & Down & 0.03059 \\
\hline AT1G07340 & STP2 & SUGAR TRANSPORTER 2 & 39.175 & 27.415 & 0.700 & Down & 0.02665 \\
\hline AT3G47440 & TIP5;1 & TONOPLAST INTRINSIC PROTEIN 5;1 & 3.16325 & 1.8435 & 0.583 & Down & 0.09475 \\
\hline AT4G35420 & TKPR1 & TETRAKETIDE ALPHA-PYRONE REDUCTASE 1 & 265 & 163.175 & 0.616 & Down & 0.009035 \\
\hline AT1G68540 & TKPR2 & TETRAKETIDE ALPHA-PYRONE REDUCTASE 2 & 18.45 & 12.1625 & 0.659 & Down & 0.05092 \\
\hline AT4G12920 & UND & UNDEAD & 11.3435 & 6.2965 & 0.555 & Down & 0.04017 \\
\hline AT4G26440 & WRKY34 & WRKY DNA-BINDING PROTEIN 34 & 7.033 & 4.24575 & 0.604 & Down & 0.009744 \\
\hline AT2G35070 & & transmembrane protein;(source:Araport11) & 8.2975 & 4.489 & 0.541 & Down & 0.01696 \\
\hline
\end{tabular}


TABLE 2 | (Continued)

\begin{tabular}{|c|c|c|c|c|c|c|c|}
\hline \multirow[t]{2}{*}{ ID } & \multirow[t]{2}{*}{ Gene symbol } & \multirow[t]{2}{*}{ Gene name/description } & \multicolumn{2}{|c|}{ FPKM } & \multirow{2}{*}{$\frac{\text { Fold change }}{(\text { rdp1-3/WT) }}$} & \multirow{2}{*}{ Up/Down } & \multirow[t]{2}{*}{ FDR } \\
\hline & & & WT & rdp1-3 & & & \\
\hline AT3G10470 & & C2H2-type zinc finger family protein;(source:Araport11) & 1.9 & 0.54983 & 0.289 & Down & 0.06933 \\
\hline AT3G28780 & & transmembrane protein, putative (DUF1216);(source:Araport11) & 127.82 & 40.83 & 0.319 & Down & 0.00002049 \\
\hline \multicolumn{8}{|c|}{ Twenty-three genes directly regulated by AMS (Xu et al., 2014) } \\
\hline AT4G14080 & MEE48 & MATERNAL EFFECT EMBRYO ARREST 48 & 445.28 & 249.95 & 0.561 & Down & 0.00152 \\
\hline AT4G20050 & QRT3 & QUARTET 3 & 68.46 & 43.42 & 0.634 & Down & 0.004464 \\
\hline AT3G52160 & KCS15 & $\begin{array}{l}\text { 3-KETOACYL-COA } \\
\text { SYNTHASE } 15\end{array}$ & 47.29 & 35.54 & 0.752 & n.s. & 0.1359 \\
\hline AT5G49070 & KCS21 & $\begin{array}{l}\text { O3-KETOACYL-COA } \\
\text { SYNTHASE } 21\end{array}$ & 8.12 & 8.72 & 1.074 & n.s. & 0.8009 \\
\hline AT1G71160 & KCS7 & $\begin{array}{l}\text { O3-KETOACYL-COA } \\
\text { SYNTHASE } 7\end{array}$ & 14.41 & 12.24 & 0.850 & n.s. & 0.4758 \\
\hline AT3G51590 & LTP12 & LIPID TRANSFER PROTEIN 12 & 2847.00 & 1431.10 & 0.503 & Down & 0.00206 \\
\hline AT1G66850 & & LTP family protein & 1532.75 & 815.58 & 0.532 & Down & 0.00003572 \\
\hline AT5G62080 & & LTP family protein & 519.88 & 285.28 & 0.549 & Down & 0.001196 \\
\hline AT3G13220 & ABCG26 & ATP-Binding Cassette G subfamily 26 & 32.95 & 24.17 & 0.734 & Down & 0.07096 \\
\hline AT4G34850 & PKSB/LAP5 & POLYKETIDE SYNTHASE B/LESS ADHESIVE POLLEN 5 & 95.72 & 67.72 & 0.707 & Down & 0.09108 \\
\hline AT4G35420 & TKPR1 & TETRAKETIDE ALPHA-PYRONE REDUCTASE 1 & 265.00 & 163.18 & 0.616 & Down & 0.009035 \\
\hline AT4G00040 & $\mathrm{CHS}$ & Chalcone and stilbene synthase family protein & 82.51 & 55.72 & 0.675 & Down & 0.01389 \\
\hline AT1G75920 & EXL5 & EXTRACELLULAR LIPASE 5 & 54.14 & 27.89 & 0.515 & Down & 0.000005513 \\
\hline AT1G75910 & EXL4 & EXTRACELLULAR LIPASE 4 & 408.30 & 210.20 & 0.515 & Down & 0.000004671 \\
\hline AT1G75930 & EXL6 & EXTRACELLULAR LIPASE 6 & 244.13 & 120.38 & 0.493 & Down & $7.601 e-07$ \\
\hline AT1G06990 & & GDSL-like Lipase/Acylhydrolase superfamily protein & 32.87 & 19.16 & 0.583 & Down & 0.003785 \\
\hline AT1G69500 & CYP704B1 & $\begin{array}{l}\text { CYTOCHROME P450, FAMILY } \\
704, \text { SUBFAMILY B, } \\
\text { POLYPEPTIDE } 1\end{array}$ & 14.82 & 11.20 & 0.756 & n.s. & 0.219 \\
\hline AT1G01280 & CYP703A2 & CYTOCHROME P450, FAMILY 703, SUBFAMILY A, POLYPEPTIDE 2 & 26.20 & 18.22 & 0.695 & Down & 0.06944 \\
\hline AT1G74540 & CYP98A8 & CYTOCHROME P450, FAMILY 98, SUBFAMILY A, POLYPEPTIDE 8 & 40.58 & 27.55 & 0.679 & Down & 0.02009 \\
\hline AT1G74550 & CYP98A9 & CYTOCHROME P450, FAMILY 98, SUBFAMILY A, POLYPEPTIDE 9 & 55.92 & 37.84 & 0.677 & Down & 0.01419 \\
\hline AT1G13140 & CYP86C3 & CYTOCHROME P450, FAMILY 86, SUBFAMILY C, POLYPEPTIDE 3 & 59.11 & 43.66 & 0.739 & Down & 0.0959 \\
\hline AT5G07520 & GRP18 & GLYCINE-RICH PROTEIN 18 & 63.58 & 31.91 & 0.502 & Down & $1.898 \mathrm{e}-07$ \\
\hline AT5G07550 & GRP19 & GLYCINE-RICH PROTEIN 19 & 5613.75 & 2498.00 & 0.445 & Down & 0.0002736 \\
\hline
\end{tabular}

The genes highlighted in blue are downregulated.

assembly" and "Ribosomal large subunit biosynthesis") in the transcriptome analysis of $r d p 1-3$. We found that $23.0 \%$ of the genes involved in the ribosomal large subunit were significantly differentially expressed (33 out of 143 genes), with most of them being upregulated (32 out of 33 genes) (Table 1). Conversely, only $8.2 \%$ of the genes involved in the ribosomal small subunit were significantly different (8 out of 98 genes). Furthermore, S222L was the only nonsynonymous mutation in the mapped region revealed by the two complementation tests; thus, it was a candidate causal mutation for the pollen-number phenotype (as discussed above). Moreover, this amino acid position was shown to be important for the cellular localization and ribosomal function of human RDP1 (Michalec-Wawiorka et al., 2015). Taken together, our results strongly support the contention that RDP1 of $A$. thaliana functions in the biogenesis of the ribosomal large subunit as a yeast Mrt4 homolog (Lo et al., 2010; Michalec-Wawiorka et al., 2015; Greber, 2016; Saez-Vasquez and Delseny, 2019).

\section{Regulation of the Expression of Pollen Development Genes in the Mutant of RDP1}

The mechanisms underlying where and how RDP1 affects the expression of bHLH transcriptional factor genes, such as AMS, bHLH010, and bHLH089, remain unknown. Previous studies have reported that the spatial expression pattern of these transcriptional factor genes and that of $R D P 1$ overlap partly in the same cell types (Zhu et al., 2011; Zhu et al., 2015; Tsuchimatsu et al., 2020). Real-time PCR, promoterGUS assay, and in situ hybridization studies have suggested that $R D P 1$ is expressed broadly and strongly in proliferating cells, including sporogenous cells (Tsuchimatsu et al., 2020). A transcriptome study of dissected tissues also demonstrated that RDP1 is expressed in both tapetal cells and the pollen lineage (microspore, bicellular, and tricellular stages) (Honys and Twell, 2004; Li et al., 2017). The expressions of bHLHO10 and bHLH089 were detected in both microspores and tapetum 
cells via in situ hybridization (Zhu et al., 2015). Moreover, in situ hybridization suggested that $A M S$ is expressed in both tapetum cells and microspores (Zhu et al., 2011), although the defect of ams mutants is primarily found in surrounding tapetum cells that provide pollen wall to the germ lines (Xu et al., 2010). These expression data suggest that these bHLH genes are co-expressed with $R D P 1$ in tapetum and microspore cells. It is reported that the feedback regulation of bHLH transcription factor genes (DYT1, bHLH010, bHLH089, and bHLH091) occurs during anther development (Cui et al., 2016). Because this feedback regulation requires the synthesis of the protein encoded by each gene, RDP1 may contribute to the expression of these genes through translation by ribosomes. This hypothesis was supported by the observation that two of these genes (bHLHO1O and bHLH089) were downregulated in the rdp1-3 mutant. We also considered the possibility that the transcriptional reduction of these bHLH genes in tapetal cells has an indirect effect on tapetum development via the microspore reduction afforded by RDP1 mutation. However, this is unlikely because the expression patterns of other genes that are essential for tapetum development, such as DYT1, $T P D 1$, and MS188, were not affected in $r d p 1-3$. We could not exclude the possibility that the aberrant translational machinery of rdp1-3 may affect the mRNA abundance, or of downstream transcription independent from the feedback (Tiruneh et al., 2013; Beine-Golovchuk et al., 2018; Cheong et al., 2021) or multi-functionalization of RDP1 independent from the ribosome. Further detailed analyses are required to evaluate this scenario. In addition, previous transcriptome data obtained for the bhlh triple mutant (bhlh010/bhlh089/bhlh091) (Zhu et al., 2015) did not show an obvious transcriptional difference for ribosomal genes, including $R D P 1$, suggesting that $R D P 1$ or ribosome function is not located downstream of the bHLH gene regulatory network.

\section{The Reduction in Pollen Number and Slower Growth Observed in the rdp1-3 Mutant Are Possibly Caused by Ribosomal Specialization}

The ribosome is a protein biosynthesis machinery that is present in all living cells. Although the ribosome has been considered a solid component common to all cells, organspecific phenotypes are revealed from mutant analysis of ribosome and ribosome-biogenesis genes in yeast, humans, and plants. This ribosome specialization is now suggested as not being a rare phenomenon (Martinez-Seidel et al., 2020; Norris et al., 2021). Reproductive defects of ribosomal mutants were observed, such as reduced stamen number, smaller pollen size, slower pollen tube elongation, and reduced functional ovules (Stirnberg et al., 2012; Luo et al., 2020). RDP1 is considered a homolog of yeast Mrt4, which is a key component for the $60 \mathrm{~S}$ ribosomal assembly. We found that the expression level of $R D P 1$ varied among different cell types and was higher in proliferating cells (Tsuchimatsu et al., 2020). Natural variants under selection supported the critical role of $R D P 1$ in adaptive evolution (Tsuchimatsu et al., 2020). Furthermore, a nonfunctional RDP1 mutant exhibited reduced pollen number and slower growth speed. These data support the idea that the high expression level of $R D P 1$ is required to respond to the high demand of ribosome production in proliferation cells. Interestingly, Tsuchimatsu et al. (2020) reported that quantitative complementation test with Uod-1 and Bor-1 showed allelic differences for pollen number but not growth speed (rosette size and bolting/flowering date). This result may suggest the ribosome specialization for pollen number. At first glance, this may be similar to cytoplasmic sterility, where the reduction of mitochondrial function results in pollen-specific phenotype, where high energy consumption may be necessary (Warmke and Lee, 1978; Chase, 2007). However, our transcriptome data does not support this idea because there was no GO term "mitochondria" in down-regulated genes. Further analysis of $R D P 1$ will provide clues regarding how ribosome specialization affects the pollen number regulation.

\section{CONCLUSION}

A previous study showed that natural variation of the RDP1 gene was responsible for pollen number in A. thaliana. Here, we showed that the standard accession Col-0 had a natural variant that reduced pollen number. Moreover, the frameshift mutant $r d p 1-3$ in the Col-0 background confirmed pleiotropic effects, including that on flowering time. A transcriptome analysis of the rdp1-3 mutant suggested the function of RDP1 in ribosome assembly and pollen development. These data support the specialized function of the ribosome in pollen development.

\section{DATA AVAILABILITY STATEMENT}

The datasets presented in this study can be found in online repositories. The names of the repository/repositories and accession number(s) can be found in the Supplementary Table 1.

\section{AUTHOR CONTRIBUTIONS}

HK, TT, MY, and KKS planned and designed the study and wrote the manuscript. HK and MY performed the experiments. HK and TT analyzed the data and drew the figures. MH managed the sequencing data. All authors contributed to the article and approved the submitted version.

\section{FUNDING}

This work was supported by a Swiss National Science Foundation grant 31003A_182318 to KKS, by MEXT KAKENHI (Grant Numbers $16 \mathrm{H} 06469$ to KKS, and $17 \mathrm{H} 05833$ and $19 \mathrm{H} 04851$ to TT), JSPS KAKENHI (Grant Number $19 \mathrm{~K} 05976$ to HK), the University Research Priority Program (URPP) Evolution in Action of the University of Zurich, and a JST CREST JPMJCR16O3 to KKS. 


\section{ACKNOWLEDGMENTS}

We thank Aluri Sirisha (Functional Genomics Center Zurich) for technical support in the transcriptome analysis, and Naoto-Benjamin Hamaya (University of Zurich) and Daisuke Maruyama (Yokohama City University) for valuable suggestions.

\section{SUPPLEMENTARY MATERIAL}

The Supplementary Material for this article can be found online at: https://www.frontiersin.org/articles/10.3389/fpls.2021. 768584/full\#supplementary-material

Supplementary Figure 1 | Nucleic acid comparison of the RDP1 region from Uod-1, Col-0, and Bor-4. The green and yellow boxes indicate mRNA and coding regions, respectively. Each annotation was defined by NCBI information (NM_102335.4).

Supplementary Figure 2 | Traditional transgenic complementation test of pollen number per flower in the rdp1-3 background. No significant difference was detected in each case in line-wise (A) and in allele-wise (B) analyses ( $n=269$ (RDP1/RDP1), 908 (rdp1-3/rdp1-3 +

\section{REFERENCES}

Alvarez-Buylla, E. R., Benitez, M., Corvera-Poire, A., Chaos Cador, A., de Folter, S., Gamboa de Buen, A., et al. (2010). Flower development. Arabidopsis Book 8:e0127. doi: 10.1199/tab.0127

Barakat, A., Szick-Miranda, K., Chang, I. F., Guyot, R., Blanc, G., Cooke, R., et al. (2001). The organization of cytoplasmic ribosomal protein genes in the Arabidopsis genome. Plant Physiol. 127, 398-415. doi: 10.1104/pp.010265

Beine-Golovchuk, O., Firmino, A. A. P., Dabrowska, A., Schmidt, S., Erban, A., Walther, D., et al. (2018). Plant temperature acclimation and growth rely on cytosolic ribosome biogenesis factor homologs. Plant Physiol. 176, 2251-2276. doi: $10.1104 / p p \cdot 17.01448$

Boeven, P. H., Longin, C. F., Leiser, W. L., Kollers, S., Ebmeyer, E., and Wurschum, T. (2016). Genetic architecture of male floral traits required for hybrid wheat breeding. Theor. Appl. Genet. 129, 2343-2357. doi: 10.1007/s00122-016-2771-6

Chase, C. D. (2007). Cytoplasmic male sterility: a window to the world of plant mitochondrial-nuclear interactions. Trends Genet. 23, 81-90. doi: 10.1016/j.tig. 2006.12.004

Chen, W., Lv, M., Wang, Y., Wang, P. A., Cui, Y., Li, M., et al. (2019). BES1 is activated by EMS1-TPD1-SERK1/2-mediated signaling to control tapetum development in Arabidopsis thaliana. Nat. Commun. 10:4164. doi: 10.1038/ s41467-019-12118-4

Cheong, B. E., Beine-Golovchuk, O., Gorka, M., Ho, W. W. H., Martinez-Seidel, F., Firmino, A. A. P., et al. (2021). Arabidopsis REI-LIKE proteins activate ribosome biogenesis during cold acclimation. Sci. Rep. 11:2410. doi: 10.1038/s41598-02181610-z

Clough, S. J., and Bent, A. F. (1998). Floral dip: a simplified method for Agrobacterium-mediated transformation of Arabidopsis thaliana. Plant J. 16, 735-743. doi: 10.1046/j.1365-313x.1998.00343.x

Creff, A., Sormani, R., and Desnos, T. (2010). The two Arabidopsis RPS6 genes, encoding for cytoplasmic ribosomal proteins S6, are functionally equivalent. Plant Mol. Biol. 73, 533-546. doi: 10.1007/s11103-010-9639-y

Cui, J., You, C. J., Zhu, E. G., Huang, Q., Ma, H., and Chang, F. (2016). Feedback regulation of DYT1 by interactions with downstream bHLH factors promotes DYT1 nuclear localization and anther development. Plant Cell 28, 1078-1093. doi: 10.1105/tpc.15.00986

Feng, B. M., Lu, D. H., Ma, X., Peng, Y. B., Sun, Y. J., Ning, G., et al. (2012). Regulation of the Arabidopsis anther transcriptome by DYT1 for pollen development. Plant J. 72, 612-624. doi: 10.1111/j.1365-313X.2012.05104.x

Feng, X., and Dickinson, H. G. (2010). Tapetal cell fate, lineage and proliferation in the Arabidopsis anther. Development 137, 2409-2416. doi: 10.1242/dev.049320

Ferguson, A. C., Pearce, S., Band, L. R., Yang, C. Y., Ferjentsikova, I., King, J., et al. (2017). Biphasic regulation of the transcription factor
$\left.R D P 1^{\mathrm{Col}-0}\right), 932$ (rdp1-3/rdp1-3 + RDP1Mz-0), 713 (rdp1-3/rdp1-3 + $\left.R D P 1^{\text {Bor }-4}\right)$, and $788($ rdp1-3/rdp1-3 + RDP1Alyrata $\left.)\right)$. Boxplots show center line: median; box limits: upper and lower quartiles; dots: outliers.

Supplementary Figure 3 | Pollen number from four genotypes of quantitative complementation test between Bor-4 and Col-0 accession. Sample numbers are shown above the plots. Boxplots show center line: median; box limits: upper and lower quartiles; whiskers: not greater than 1.5 times the interquartile range; point: outlier. Violin shape corresponds to the density of data.

Supplementary Table 1 | Sample information and read numbers of transcriptome analysis in this study.

Supplementary Table 2 | List of differentially expressed genes (3020 genes) between wild-type and rdp1-3 plants.

Supplementary Table 3 | TOP500 GO enrichment list (Biological process) for upregulated DEGs in rdp1-3 plants.

Supplementary Table 4 | Complete GO enrichment list (Biological process) for downregulated DEGs in rdp1-3 plants.

Supplementary Video 1 | Time-lapse movie of RDP1/RDP1 (left) and rdp1-3/rdp1-3 (right) plants. Photos were taken every hour from 10h00 to 19h00 for 39 days under the long-day condition.

ABORTED MICROSPORES (AMS) is essential for tapetum and pollen development in Arabidopsis. New Phytol. 213, 778-790. doi: 10.1111/nph. 14200

Franco-Mora, O., Tanabe, K., Tamura, F., and Itai, A. (2005). Effects of putrescine application on fruit set in 'Housui' Japanese pear (Pyrus pyrifolia Nakai). Sci. Hortic. 104, 265-273. doi: 10.1016/j.scienta.2004.10.005

Fujikura, U., Horiguchi, G., Ponce, M. R., Micol, J. L., and Tsukaya, H. (2009) Coordination of cell proliferation and cell expansion mediated by ribosomerelated processes in the leaves of Arabidopsis thaliana. Plant J. 59, 499-508. doi: 10.1111/j.1365-313X.2009.03886.x

Ge, S. X., Jung, D., and Yao, R. (2020). ShinyGO: a graphical gene-set enrichment tool for animals and plants. Bioinformatics 36, 2628-2629. doi: 10.1093/ bioinformatics/btz931

Greber, B. J. (2016). Mechanistic insight into eukaryotic 60S ribosomal subunit biogenesis by cryo-electron microscopy. RNA 22, 1643-1662. doi: 10.1261/rna. 057927.116

Hatakeyama, M., Opitz, L., Russo, G., Qi, W. H., Schlapbach, R., and Rehrauer H. (2016). SUSHI: an exquisite recipe for fully documented, reproducible and reusable NGS data analysis. BMC Bioinformatics 17:228. doi: 10.1186/s12859016-1104-8

Honys, D., and Twell, D. (2004). Transcriptome analysis of haploid male gametophyte development in Arabidopsis. Genome Biol. 5:R85. doi: 10.1186/gb2004-5-11-r85

Horiguchi, G., Molla-Morales, A., Perez-Perez, J. M., Kojima, K., Robles, P., Ponce, M. R., et al. (2011). Differential contributions of ribosomal protein genes to Arabidopsis thaliana leaf development. Plant J. 65, 724-736. doi: 10.1111/j. 1365-313X.2010.04457.x

Hummel, M., Dobrenel, T., Cordewener, J., Davanture, M., Meyer, C., Smeekens, S., et al. (2015). Proteomic LC-MS analysis of Arabidopsis cytosolic ribosomes: identification of ribosomal protein paralogs and re-annotation of the ribosomal protein genes. J. Proteom. 128, 436-449. doi: 10.1016/j.jprot.2015.07.004

Kakui, H., Yamazaki, M., Hamaya, N.-B., and Shimizu, K. K. (2020). "Pollen grain counting using a cell counter," in Pollen and Pollen Tube Methods, ed A. Geitmann (New York: Springer), 1-11. doi: 10.1007/978-1-0716-0672-8

Kakui, H., Yamazaki, M., and Shimizu, K. K. (2021). PRIMA: a rapid and cost-effective genotyping method to detect single-nucleotide differences using probe-induced heteroduplexes. Sci. Rep. 11:20741. doi: 10.1038/s41598-02199641-x

Langer, S. M., Longin, C. F. H., and Wurschum, T. (2014). Phenotypic evaluation of floral and flowering traits with relevance for hybrid breeding in wheat (Triticum aestivum L.). Plant Breed. 133, 433-441. doi: 10.1111/pbr.12192

Li, D. D., Xue, J. S., Zhu, J., and Yang, Z. N. (2017). Gene regulatory network for tapetum development in Arabidopsis 
thaliana. Front. Plant Sci. 8:1559. doi: 10.3389/fpls.2017.01 559

Lo, K. Y., Li, Z., Bussiere, C., Bresson, S., Marcotte, E. M., and Johnson, A. W. (2010). Defining the pathway of cytoplasmic maturation of the $60 \mathrm{~S}$ ribosomal subunit. Mol. Cell 39, 196-208. doi: 10.1016/j.molcel.2010.06.018

Lou, Y., Zhou, H. S., Han, Y., Zeng, Q. Y., Zhu, J., and Yang, Z. N. (2018). Positive regulation of AMS by TDF1 and the formation of a TDF1-AMS complex are required for anther development in Arabidopsis thaliana. New Phytol. 217, 378-391. doi: 10.1111/nph.14790

Luo, A., Zhan, H. D., Zhang, X. C., Du, H. W., Zhang, Y. B., and Peng, X. B. (2020). Cytoplasmic ribosomal protein L14B is essential for fertilization in Arabidopsis. Plant Sci. 292:110394. doi: 10.1016/j.plantsci.2019.110394

Ma, X., Feng, B. M., and Ma, H. (2012). AMS-dependent and independent regulation of anther transcriptome and comparison with those affected by other Arabidopsis anther genes. BMC Plant Biol. 12:23. doi: 10.1186/1471-2229-12-23

Martinez-Seidel, F., Beine-Golovchuk, O., Hsieh, Y. C., and Kopka, J. (2020). Systematic review of plant ribosome heterogeneity and specialization. Front. Plant Sci. 11:948. doi: 10.3389/fpls.2020.00948

Michalec, B., Krokowski, D., Grela, P., Wawiorka, L., Sawa-Makarska, J., Grankowski, N., et al. (2010). Subcellular localization of ribosomal P0-like protein MRT4 is determined by its N-terminal domain. Int. J. Biochem. Cell Biol. 42, 736-748. doi: 10.1016/j.biocel.2010.01.011

Michalec-Wawiorka, B., Wawiorka, L., Derylo, K., Krokowski, D., Boguszewska, A., Molestak, E., et al. (2015). Molecular behavior of human Mrt4 protein, MRTO4, in stress conditions is regulated by its C-terminal region. Int. J. Biochem. Cell Biol. 69, 233-240. doi: 10.1016/j.biocel.2015.10.018

Norris, K., Hopes, T., and Aspden, J. L. (2021). Ribosome heterogeneity and specialization in development. Wiley Interdiscip. Rev. RNA 12:e1644. doi: 10. 1002/wrna.1644

Oka, H. I., and Morishima, H. (1967). Variations in the breeding systems of a wild rice, Oryza perennis. Evolution 21, 249-258. doi: 10.1111/j.1558-5646.1967. tb00153.x

Podobnik, M., Frohnhofer, H. G., Dooley, C. M., Eskova, A., Nusslein-Volhard, C., and Irion, U. (2020). Evolution of the potassium channel gene Kcnj13 underlies colour pattern diversification in Danio fish. Nat. Commun. 11:6230. doi: 10.1038/s41467-020-20021-6

R Core Team (2013). R: A Language and Environment for Statistical Computing. Vienna: R Foundation for Statistical Computing.

Rodriguez-Mateos, M., Abia, D., Garcia-Gomez, J. J., Morreale, A., de la Cruz, J., Santos, C., et al. (2009a). The amino terminal domain from Mrt4 protein can functionally replace the RNA binding domain of the ribosomal P0 protein. $\mathrm{Nucl}$. Acids Res. 37, 3514-3521. doi: 10.1093/nar/gkp209

Rodriguez-Mateos, M., Garcia-Gomez, J. J., Francisco-Velilla, R., Remacha, M., de la Cruz, J., and Ballesta, J. P. (2009b). Role and dynamics of the ribosomal protein $\mathrm{P} 0$ and its related trans-acting factor Mrt4 during ribosome assembly in Saccharomyces cerevisiae. Nucl. Acids Res. 37, 7519-7532. doi: 10.1093/nar/ gkp806

Saez-Vasquez, J., and Delseny, M. (2019). Ribosome biogenesis in plants: from functional $45 \mathrm{~S}$ ribosomal DNA organization to ribosome assembly factors. Plant Cell 31, 1945-1967. doi: 10.1105/tpc.18.00874

Salih, K. J., Duncan, O., Li, L., Trosch, J., and Millar, A. H. (2020). The composition and turnover of the Arabidopsis thaliana $80 \mathrm{~S}$ cytosolic ribosome. Biochem. J. 477, 3019-3032. doi: 10.1042/BCJ20200385

Sanders, P. M., Bui, A. Q., Weterings, K., McIntire, K. N., Hsu, Y. C., Lee, P. Y., et al. (1999). Anther developmental defects in Arabidopsis thaliana male-sterile mutants. Sex. Plant Reprod. 11, 297-322. doi: 10.1007/s004970050158

Schmidt, S., Dethloff, F., Beine-Golovchuk, O., and Kopka, J. (2013). The REIL1 and REIL2 proteins of Arabidopsis thaliana are required for leaf growth in the cold. Plant Physiol. 163, 1623-1639. doi: 10.1104/pp.113.223925

Shimada, T. L., Shimada, T., and Hara-Nishimura, I. (2010). A rapid and nondestructive screenable marker, FAST, for identifying transformed seeds of Arabidopsis thaliana. Plant J. 61, 519-528. doi: 10.1111/j.1365-313X.2009. 04060.x

Shimizu, K. K., and Tsuchimatsu, T. (2015). Evolution of selfing: recurrent patterns in molecular adaptation. Annu. Rev. Ecol. Evol. Syst. 46, 593-622. doi: 10.1146/ annurev-ecolsys-112414-054249

Smyth, D. R., Bowman, J. L., and Meyerowitz, E. M. (1990). Early flower development in Arabidopsis. Plant Cell 2, 755-767. doi: 10.1105/tpc.2.8.755

Sorensen, A. M., Krober, S., Unte, U. S., Huijser, P., Dekker, K., and Saedler, H. (2003). The Arabidopsis ABORTED MICROSPORES (AMS) gene encodes a
MYC class transcription factor. Plant J. 33, 413-423. doi: 10.1046/j.1365-313x. 2003.01644.x

Steinmetz, L. M., Sinha, H., Richards, D. R., Spiegelman, J. I., Oefner, P. J., McCusker, J. H., et al. (2002). Dissecting the architecture of a quantitative trait locus in yeast. Nature 416, 326-330. doi: 10.1038/416326a

Stern, D. L. (1998). A role of Ultrabithorax in morphological differences between Drosophila species. Nature 396, 463-466. doi: 10.1038/24863

Stern, D. L. (2014). Identification of loci that cause phenotypic variation in diverse species with the reciprocal hemizygosity test. Trends Genet. 30, 547-554. doi: 10.1016/j.tig.2014.09.006

Stirnberg, P., Liu, J. P., Ward, S., Kendall, S. L., and Leyser, O. (2012). Mutation of the cytosolic ribosomal protein-encoding RPS10B gene affects shoot meristematic function in Arabidopsis. BMC Plant Biol. 12:160. doi: 10. 1186/1471-2229-12-160

Tiruneh, B. S., Kim, B. H., Gallie, D. R., Roy, B., and von Arnim, A. G. (2013). The global translation profile in a ribosomal protein mutant resembles that of an eIF3 mutant. BMC Biol. 11:123. doi: 10.1186/1741-7007-11-123

Tsuchimatsu, T., Kakui, H., Yamazaki, M., Marona, C., Tsutsui, H., Hedhly, A., et al. (2020). Adaptive reduction of male gamete number in the selfing plant Arabidopsis thaliana. Nat. Commun. 11:2885. doi: 10.1038/s41467-020-16679-7

Tsutsui, H., and Higashiyama, T. (2017). pKAMA-ITACHI vectors for highly efficient CRISPR/Cas9-mediated gene knockout in Arabidopsis thaliana. Plant Cell Physiol. 58, 46-56. doi: 10.1093/pcp/pcw191

Turner, T. L. (2014). Fine-mapping natural alleles: quantitative complementation to the rescue. Mol. Ecol. 23, 2377-2382. doi: 10.1111/mec.12719

Walbot, V., and Egger, R. L. (2016). Pre-meiotic anther development: cell fate specification and differentiation. Annu. Rev. Plant Biol. 67, 365-395. doi: 10. 1146/annurev-arplant-043015-111804

Wang, K., Guo, Z. L., Zhou, W. T., Zhang, C., Zhang, Z. Y., Lou, Y., et al. (2018). The regulation of sporopollenin biosynthesis genes for rapid pollen wall formation. Plant Physiol. 178, 283-294. doi: 10.1104/pp.18.00219

Warmke, H. E., and Lee, S.-L. J. (1978). Pollen abortion in T cytoplasmic malesterile corn (Zea mays): a suggested mechanism. Science 200, 561-563. doi: $10.1126 /$ science.200.4341.561

Xu, J., Ding, Z., Vizcay-Barrena, G., Shi, J., Liang, W., Yuan, Z., et al. (2014). ABORTED MICROSPORES acts as a master regulator of pollen wall formation in Arabidopsis. Plant Cell 26, 1544-1556. doi: 10.1105/tpc.114.122986

Xu, J., Yang, C., Yuan, Z., Zhang, D., Gondwe, M. Y., Ding, Z., et al. (2010). The ABORTED MICROSPORES regulatory network is required for postmeiotic male reproductive development in Arabidopsis thaliana. Plant Cell 22, 91-107. doi: $10.1105 /$ tpc. 109.071803

Xue, J. S., Yao, C., Xu, Q. L., Sui, C. X., Jia, X. L., Hu, W. J., et al. (2021). Development of the middle layer in the anther of Arabidopsis. Front. Plant Sci. 12:634114. doi: 10.3389/fpls.2021.634114

Zhu, E. G., You, C. J., Wang, S. S., Cui, J., Niu, B. X., Wang, Y. X., et al. (2015). The DYT1-interacting proteins bHLH010, bHLH089 and bHLH091 are redundantly required for Arabidopsis anther development and transcriptome. Plant J. 83, 976-990. doi: 10.1111/tpj. 12942

Zhu, J., Lou, Y., Xu, X., and Yang, Z. N. (2011). A genetic pathway for tapetum development and function in Arabidopsis. J. Integr. Plant Biol. 53, 892-900. doi: 10.1111/j.1744-7909.2011.01078.x

Conflict of Interest: The authors declare that the research was conducted in the absence of any commercial or financial relationships that could be construed as a potential conflict of interest.

Publisher's Note: All claims expressed in this article are solely those of the authors and do not necessarily represent those of their affiliated organizations, or those of the publisher, the editors and the reviewers. Any product that may be evaluated in this article, or claim that may be made by its manufacturer, is not guaranteed or endorsed by the publisher.

Copyright (c) 2022 Kakui, Tsuchimatsu, Yamazaki, Hatakeyama and Shimizu. This is an open-access article distributed under the terms of the Creative Commons Attribution License (CC BY). The use, distribution or reproduction in other forums is permitted, provided the original author(s) and the copyright owner(s) are credited and that the original publication in this journal is cited, in accordance with accepted academic practice. No use, distribution or reproduction is permitted which does not comply with these terms. 